\title{
Indication of Pancreas Transplantation (Donor and Recipient)
}

\author{
Takashi Kenmochi and Duck-Jong Han
}

\section{Donor}

See the Living Donor chapter.

\section{Recipient}

\section{General}

See the Living Donor chapter.

\section{Korea}

The indication of $\mathrm{ABO}$ incompatible living donor pancreas transplantation is the same as the $\mathrm{ABO}$ compatible living donor pancreas transplantation (See the Living Donor chapter). However, when IgG titer below 1:64 or IgM titer below 1:8 cannot be achieved especially in blood O-type recip- ient following desensitization, operation was held regardless of initial anti-ABO titer.

\section{Japan}

Indication for the recipient of ABOi LDPT is the same as ABO compatible LDPT as shown in Chapter 10. However, we indicated ABOi LDPT only for SPK category because the biopsy of the transplanted kidney was essential for monitoring antibody-mediated rejection (AMR) due to $\mathrm{ABO}$ incompatibility. In addition, the recipient whose anti-A or -B antibodies was extremely high, which showed over 512-folds, was considered to be a relative contraindication because of the possibility of high risk of AMR. Also, the crossmatch both of $\mathrm{T}$ cell and $\mathrm{B}$ cell with flow cytometry, in addition, to direct crossmatch should be negative in the recipient.

T. Kenmochi

Fujita Health University, Toyoake, Japan

e-mail: kenmochi@fujita-hu.ac.jp

D.-J. Han $(\bowtie)$

Asan Medical Center, Seoul, Korea 